\title{
EFEITO DAS CONDIÇÕES DE PROCESSAMENTO DE CERÂMICAS DE SNO2 DOPADAS COM Sr ${ }^{2+*}$
}

Bruna de Camargo Lemos $^{1}$ Gilberto José Pereira²

\section{Resumo}

O objetivo deste trabalho compreende a realização da síntese de $\mathrm{SnO}_{2}$ dopado com aditivo $\mathrm{Sr}^{2}$ contendo diferentes quantidades molares de $\mathrm{SrO}(\mathrm{X}) \mathrm{SnO}_{2}+(1-\mathrm{X}) \mathrm{SrO}$, em que o cátion $\mathrm{Sr}^{2+}$ foi introduzido através do nitrato de estrôncio variando de 5 à $50 \%$ em mol para uma mistura tanto em meio aquoso quanto em meio alcoólico, seguida da calcinação do pó resultante que é feita à $700^{\circ} \mathrm{C}$ com uma taxa de $10^{\circ} \mathrm{C} / \mathrm{min}$. Análises de caracterização como difração de raios $X$, área específica, calorimetria exploratória, picnometria a gás e determinação do ponto isoelétrico das suspensões aquosas foram realizadas de modo a verificar qual o agente dispersante mais apropriado para as características ácido-básicos da suspensão em questão. A partir deste determinar o potencial zeta do composto estudado através da eletroforese. Compostos intermediários a base de estanato de estanho foram identificados, e a deposição por eletroforese foi realizada com sucesso, uma vez que foi possível obter uma suspensão estável a partir dos pós cerâmicos produzidos.

Palavras-chave: Síntese; Acidobásica; Eletroforese; Potencial zeta.

\section{EFFECT OF PROCESSING CONDITIONS TO GET SNO2 CERAMICS DOPED BY $\mathrm{Sr}^{2+}$}

\section{Abstract}

The objective of this work is comprehension a synthesis of $\mathrm{SnO}_{2}$ doping with additive $\mathrm{Sr}_{2}{ }^{+}$that containing different molar quantities of $\mathrm{SrO}(\mathrm{X}) \mathrm{SnO}_{2}+(1-\mathrm{X}) \mathrm{SrO}$, wherein the cation was added using the nitrate tin ringing from 5 to $50 \%$ mol using both aqueous medium and for alcoholic medium for the mix. Then calcination was performed on the resulting powder in a $700^{\circ} \mathrm{C}$ with a rate of $10^{\circ} \mathrm{C} / \mathrm{min}$. The characterization analyses as $\mathrm{x}$-ray diffraction, specific area, scanning calorimetry, gas pycnometry and isoelectric point of aqueous suspensions were made in order to verify the most suitable dispersant for the acid-base characteristics of this suspension in question. From these analyses is possible to determine the zeta potential of the compound studied by electrophoresis. Intermediate compounds of tin stannate base were identified by electrophoresis and deposition was successfully performed.

Keywords: Synthesis; Acidobásica; Electrophoresis; Zeta potential.

1 Engenharia de materiais, estudante, departamento de Materiais, Centro universitário da FEI, São Bernardo do Campo, São Paulo, Brasil.

2 Professor orientador, Doutor, departamento de Materiais, Centro universitário da FEI, São Bernardo do Campo, São Paulo, Brasil. 


\section{INTRODUÇÃO}

O método de deposição eletroforético é utilizado industrialmente para a deposição de revestimentos ou películas sobre objetos eletricamente condutores, também é uma técnica que viabiliza a obtenção de materiais compósitos sintetizados a partir de nanopartículas em suspensão. Resultando em resistência melhorada e/ou propriedades elétricas úteis. O óxido de estanho $\left(\mathrm{SnO}_{2}\right)$ por sua vez, é um material semicondutor, com utilização em resistores elétricos e também como partes de catalizadores. O objetivo do trabalho é através da dopagem e sintetização do mesmo é possível adquirir propriedades elétricas mais abrangentes como no caso dos materiais dielétricos.

\section{$1.1 \mathrm{SnO}_{2}$}

O óxido de estanho é obtido a partir da cassiterita e possui densidade relativa 6,95 $\mathrm{g} / \mathrm{cm}^{3}$ segundo a literatura. Possui estrutura cristalina tetragonal do rutilo e é uma estrutura com coordenação 6:3, com valores de $a=4,737 \underline{\AA}$ e c $=3,185 \underline{\AA}$ [1]. Em sua forma pura, o óxido de estanho é um semicondutor do tipo $n$, que aumenta o número de portadores de cargas livre negativas (elétrons) e de gap $3,6 \mathrm{eV}$, isso acontece por ele possuir defeitos em sua estrutura cristalina. Esse defeito nada mais é do que a deficiência em oxigênio, já que a presença de $\mathrm{Sn}^{2+}$ perturba a eletronegatividade do cristal pela criação de deficiência em carga positiva (excesso de carga negativa) e tal excesso é compensado pela formação de uma vacância de oxigênio para cada ion $\mathrm{Sn}^{2+}$ formado [1]. Essa vacância é formada pela transformação de um átomo de oxigênio de um sítio da rede para o estado gasoso, essa também pode ser formada através de uma solução sólida substitucional. Vacância de oxigênio são espaços que permitem locomoção, assim conferindo condutividade que aumenta a medida que houver mais espaços (vacâncias).

\subsection{Estrôncio}

O estrôncio é encontrado na natureza nas formas de sulfato e carbonato e são considerados os mais estáveis dos elementos que constituem nitratos e carbonatos da família $2 \mathrm{~A}$ quando comparados com os oxidados. O óxido de estrôncio puro apresenta forte caráter básico quando em suspensão aquosa, e tem potencial catalítico [2].

\section{$1.3 \mathrm{SnO}_{2}+\mathrm{Sr}^{2+}$}

Existem diferentes compostos cristalinos no sistema $\mathrm{SnO}_{2}-\mathrm{Sr}\left(\mathrm{NO}_{3}\right)_{2}$, sendo os mais conhecidos $\mathrm{SrO}, \mathrm{SrSnO}_{3}, \mathrm{SnO}, \mathrm{Sn}_{3}+2 \mathrm{O}_{2}(\mathrm{OH})_{2}, \mathrm{Sr}_{5} \mathrm{Sn}_{3}, \mathrm{Sn} \mathrm{Sr}_{2}, \mathrm{SrSnO}_{3}$ e seus derivados. A adição de quantidades estequiométricas de estrôncio a partir de sais, como nitratos, podem durante a síntese de óxidos pelo precursor formarem os compostos citados dependendo das condições de síntese. $\mathrm{SrSnO}_{3}$ é o estanato de estrôncio que é um material dielétrico com estrutura perovskita considerado um semicondutor, além de possui boa estabilidade térmica e mecânica, normalmente aplicado como sensor e capacitor [3]. A reação para obtenção deste componente:

Fase estável: $\mathrm{Sn}_{(\mathrm{S})}+\mathrm{Sr}\left(\mathrm{NO}_{3}\right)_{2}(\mathrm{l})+1 / 2 \mathrm{O}_{2}(\mathrm{~g}) \rightarrow \mathrm{SrSnO}_{3}(\mathrm{~S})+2 \mathrm{NO}_{2(\mathrm{~g})}$

Fase metaestável: $\mathrm{Sn}_{(\mathrm{S})}+2 \mathrm{Sr}\left(\mathrm{NO}_{3}\right)_{2}(\mathrm{~g})+1 / 2 \mathrm{O}_{2}(\mathrm{~g}) \rightarrow \mathrm{SrSnO}_{4}(\mathrm{~S})+4 \mathrm{NO}_{2}(\mathrm{~g})$ 


\section{MATERIAIS E MÉTODOS}

\subsection{Síntese das Amostras}

A síntese dos pós metálicos é feita em primeira instância com a mistura de $5 \mathrm{~g}$ óxido de estanho $\left(\mathrm{SnO}_{2}\right)$ em pó e do percursor de nitrato de estrôncio $\left(\mathrm{Sr}\left(\mathrm{NO}_{3}\right)_{2}\right)$ em diferentes concentrações 5\%,10\%,30\%, 40\% e 50\% em volume molar. Para que haja uma melhor dispersão do material esse é adicionado à um meio líquido, entretanto para um melhor estudo utiliza-se $50 \mathrm{ml}$ em meio aquoso e alcóolico. Todas as diferentes concentrações são colocadas para homogeneizar em um moinho de bolas com agentes de moagem esféricos em torno de 5 horas. Após a homogeneização é feita a filtração a vácuo e as amostras são colocadas para secar em uma estufa por 24 horas à $120^{\circ} \mathrm{C}$. Depois de secas essas podem ser calcinadas em uma mufla por um período de 6 horas à $700^{\circ} \mathrm{C} \mathrm{com}$ taxa de aquecimento de $10{ }^{\circ} \mathrm{C} / \mathrm{min}$, entretanto metade de todos os pós obtidos não foram calcinados, justamente para se avaliar o efeito da calcinação na sintetização.

\subsection{Caracterização por Picnometria a Gás}

A determinação da densidade real dos pós de óxido de estanho puro e dopados foi determinada pelo processo de picnometria a gás hélio $(\mathrm{He})$ através do equipamento Micromeritics ACCUPYC 1340. Todos os pós foram ensaiados a 180 purgas e em compartimento de $1 \mathrm{~cm}^{3}$.

\subsection{Caracterização por Difração de Raios-X}

A fim de conhecer as fases presentes nos pós dopados é feita a análise de difração de raio- $X$, com uso do equipamento Shimadzu XRD-7000 que foi executada com os seguintes parâmetros: radiação de $\mathrm{Cu}$-Ka, com passo de 0,02 , velocidade de $0,5 \mathrm{passo} / \mathrm{segundo}$ e amplitude de varredura de 22 a $82^{\circ}$.

\subsection{Análise de DSC}

Essa análise é importante para esclarecer os resultados obtidos no DRX, como por exemplo à que temperatura ocorre o surgimento de novas fases na sintetização, assim como se essa transformação é endotérmica ou exotérmica, além de indicar temperatura de fusão do material dentre outros. No trabalho presente foi utilizado o equipamento SETSYS em uma rampa de aquecimento de $10^{\circ} \mathrm{C} / \mathrm{min}$ até $1000^{\circ} \mathrm{C}$.

\subsection{Análise de BET}

Esse ensaio é para verificar a área específica dos pós produzidos antes e após a calcinação e assim analisar qual a melhor composição que nos permita o melhor resultado. O equipamento utilizado foi da fabricante MICROMERITICS da série GEMINI VII.

\subsection{Análise ESA}

As análises de potencial zeta foram realizadas em suspensões aquosas em concentração de $1 \%$ em volume de sólidos. O ponto isoelétrico foi determinado 
com a titulação potenciométrica usando ácido nítrico $2 \mathrm{~mol} / \mathrm{L}$ e Hidróxido de amônio $2 \mathrm{~mol} / \mathrm{L}$ variando 0,05 unidades de $\mathrm{pH}$ com tempo de estabilização igual a 30s. Para análise de concentração de dispersante, foi utilizado o dispersante poliacrilato de sódio $15 \%$ em peso, com adição controlada de 20 uL a cada 30 s.

\subsection{Produção da Barbotina}

O preparo da suspensão foi através do processo de barbotina, que $10 \mathrm{~g}$ da composição de $\mathrm{SnO}_{2} 30 \% \mathrm{Sr}\left(\mathrm{NO}_{3}\right)_{2}$ meio alcoólico (isopropílico) calcinada à $700^{\circ} \mathrm{C}$ foi misturado com $6 \mathrm{ml}$ de dispersante poliacrilato de sódio, DISPEX N-40 e $1 \mathrm{ml}$ de água destilada. Em seguida a mistura ficou no agitador SILVERSON L5M durante 5 minutos.

\subsection{Deposição Eletroforética}

Neste processo é feito o carregamento elétrico das partículas sólidas da suspensão preparada por meio de um sistema que consiste em uma fonte de tensão e dois pontos de corrente o cátodo, negativo e o anodo, positivo. A deposição foi feita em eletrodos de aço que funcionam como cátodo a uma tensão constante de $10 \mathrm{~V}$. $\mathrm{O}$ tempo de permanência da suspensão sobre a tensão foi de 15", 30", 60", 90" e 180 segundos. E o comprimento das partículas aderidas no eletrodo foram medidas.

\section{RESULTADOS E DISCUSSÃO}

Após as amostras serem sintetizadas nas concentrações de $5 \%, 10 \%, 30 \%, 40 \%$ e $50 \%$ em precursor tanto para meio aquoso quanto para meio alcoólico, essas foram submetidas a análise de difração de raios- $\mathrm{X}$ e comparadas com a amostra de $\mathrm{SnO}_{2}$ pura nos mesmos meios. O resultado foi que em meio aquoso não foi verificada nenhuma mudança de cristalinidade, sendo mantida a estrutura da amostra pura. Em meio alcoólico as amostras de 5\% e 10\% apresentaram picos de difração correspondentes tanto a amostra pura como ao precursor de nitrato de estrôncio adsorvido na partícula. Para as concentrações de $30 \%$ e $50 \%$ foi verificada a formação de partículas de segunda fase correspondentes ao estanato de estrôncio e para a concentração de $40 \%$ a formação de estanato de estrôncio metaestável, ambos os compostos já citados na introdução. O gráfico da figura 1 faz uma comparação a partir de um difratograma entre a amostra pura e a amostra nas concentrações de $30 \%$ e $50 \%$ que são as que apresentaram resposta a dopagem formando a fase estável. Já o gráfico da figura 2 apresenta uma comparação da amostra pura com a amostra de $40 \%$ no qual formou a fase metaestável. 

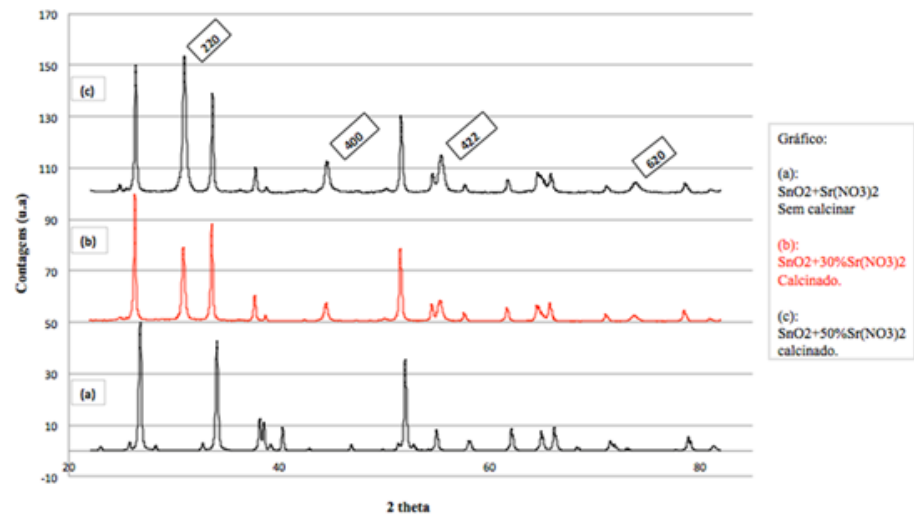

figura 1: Gráfico de comparação entre os difratograma da síntese de $\mathrm{SnO}_{2}$ com precursor de $\mathrm{Sr}\left(\mathrm{NO}_{3}\right)_{2}$ com e sem calcinar e nas proporções de $30 \%$ e $50 \%$.

Comparação gráfica entre a dopagem antes de depois de calcinada na concentrações de $40 \%$.

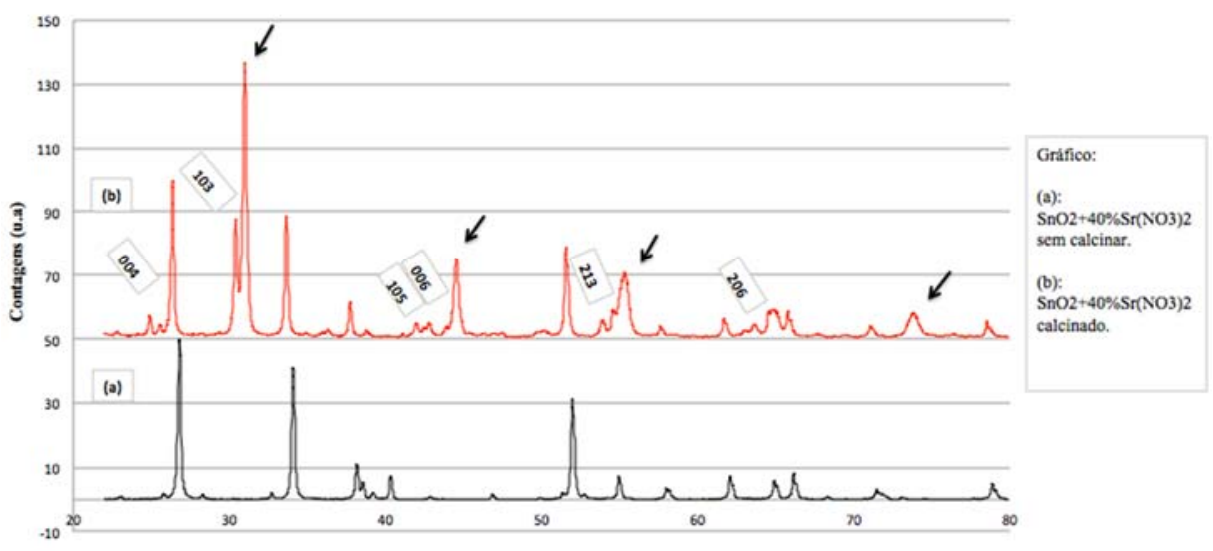

2 theta

figura 2: Gráfico de comparação entre os difratograma da síntese de $\mathrm{SnO}_{2}$ com precursor de $\mathrm{Sr}\left(\mathrm{NO}_{3}\right)_{2}$ na proporção de $40 \%$ com e sem calcinar.

A tabela 1 a seguir apresenta os valores obtidos através do ensaio de picnometria a gás, a fim de conferir as características dos pós obtidos pela síntese.

Tabela 1: Densidade das amostras obtidas pela análise de picnometria a gás

\begin{tabular}{|c|c|c|}
\hline Amostra & Antes da calcinação & Após a calcinação \\
\hline $\begin{array}{l}\text { Amostras dopadas com } 5 \% \text {, } \\
10 \%, 30 \%, 40 \% \text { e } 50 \% \text { em } \\
\text { meio aquoso. }\end{array}$ & $6,74 \mathrm{~g} / \mathrm{cm}^{3}$ & $6,67 \mathrm{~g} / \mathrm{com}^{3}$ \\
\hline $\begin{array}{l}\text { Amostras dopadas com } 5 \% \text { e } \\
10 \% \text { em meio alcoólico. }\end{array}$ & $6,74 \mathrm{~g} / \mathrm{cm}^{3}$ & $6,67 \mathrm{~g} / \mathrm{com}^{3}$ \\
\hline $\begin{array}{l}\text { Amostra dopada com } 30 \% \\
\text { em meio alcoólico. }\end{array}$ & $4,73 \mathrm{~g} / \mathrm{cm}^{3}$ & $5,66 \mathrm{~g} / \mathrm{cm}^{3}$ \\
\hline $\begin{array}{l}\text { Amostra dopada com } 40 \% \\
\text { em meio alcoólico. }\end{array}$ & $4,36 \mathrm{~g} / \mathrm{cm}^{3}$ & $5,75 \mathrm{~g} / \mathrm{cm}^{3}$ \\
\hline $\begin{array}{l}\text { Amostra dopada com } 50 \% \\
\text { em meio alcoólico. }\end{array}$ & $4,30 \mathrm{~g} / \mathrm{cm}^{3}$ & $5,83 \mathrm{~g} / \mathrm{cm}^{3}$ \\
\hline
\end{tabular}

Nota-se que houve mudança de densidade exatamente nas concentrações em que na difração de raios- $X$ apresentou mudanças em comparação a amostra pura para 
ambos os meios. Os valore de densidade que apresentaram diminuição nas concentrações de $30 \%$, 40\% e 50\% sem calcinar foram causadas devido a adsorção do precursor em sua superfície, porém as mesmas amostras calcinadas também apresentaram diminuição de densidade, mas devido a formação de partículas de segunda fase, porém com valores diferentes.

Como a proposta do trabalho é a obtenção da melhor dopagem para efeito de deposição eletroforética, essa será mais efetiva quanto maior for a área reativa, o que consequentemente são partícula de menor tamanho. Portanto a tabela 2 apresenta os resultados obtidos através do ensaio de BET, que é responsável por caracterizar a área superficial da partícula.

Tabela 2: Tabela de resultados da análise de BET.

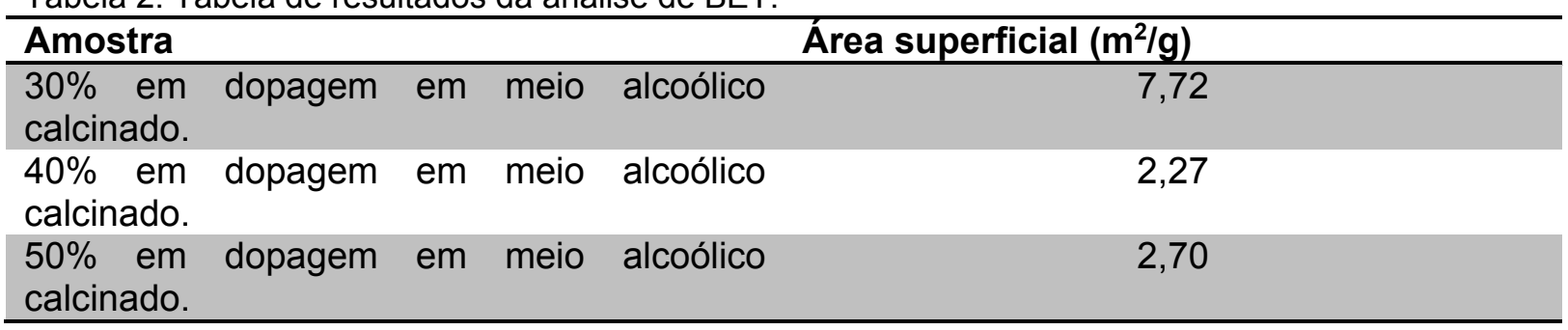

Com os resultados obtidos pelo BET das amostras dopadas que apresentaram formação de partículas de segunda fase, as amostras de $40 \%$ e $50 \%$ em precursor mostraram-se com partículas grosseiras, enquanto a amostra de $30 \%$ com partículas mais refinadas, menores. Sendo assim é possível através desse ensaio dizer que a concentração de $30 \%$ foi a que apresentou melhores características necessárias para a deposição eletroforética.

A amostra de $30 \%$ em precursor sem calcinar foi submetida a análise de DSC para verificar as transformações que o material sofre através das reações endotérmicas ou exotérmicas que ocorrem durante a calcinação, lembrando que o DSC fornece a temperatura necessária a calcinação. O gráfico da figura 3 apresenta o resultado obtido pelo DSC.

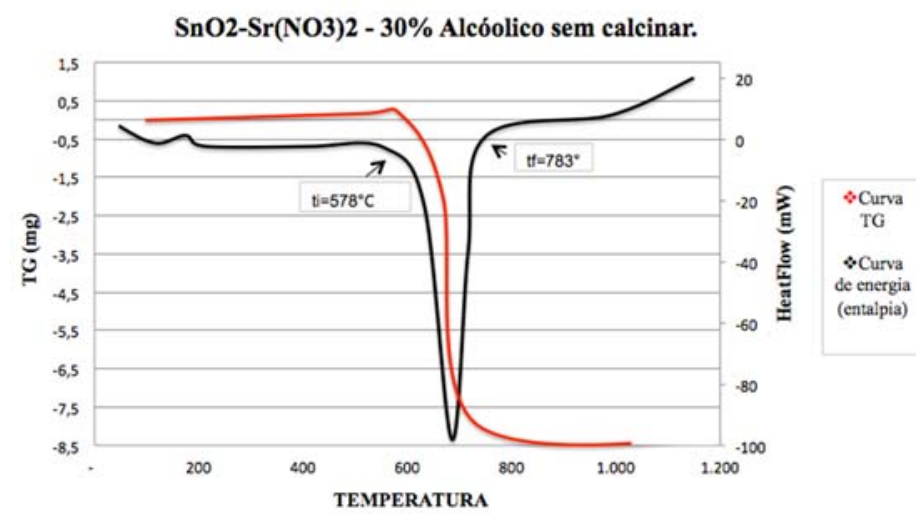

figura 3: Gráfico da análise de DSC feito no centro universitário da $\mathrm{FEI}$ da amostra de $\mathrm{SnO}_{2} 30 \%$ dopada com precursor e sem calcinar.

O gráfico de DSC obtido mostra que a uma temperatura de $578^{\circ} \mathrm{C}$ inicia-se um fenômeno de entalpia positiva, o que caracteriza uma fusão. A fusão de óxidos ocorre a aproximadamente $1000^{\circ} \mathrm{C}$, sendo assim a fusão vista no gráfico só pode ser do precursor. Isso se torna uma confirmação de que a temperatura de calcinação escolhida para o seguinte trabalho foi coerente em $700^{\circ} \mathrm{C}$, suficiente para que 0 
precursor seja adsorvido pelo pó cerâmico e funda-se ocorrendo a calcinação e coalescimento das partículas (calcinação líquida). A formação de partículas de segunda fase deve ocorrer a uma temperatura de aproximadamente $700^{\circ} \mathrm{C}$ que foi a temperatura da calcinação da amostra estudada, portanto deveria ser possível ver um pico exotérmico na análise. Entretanto este pico não foi verificado, o que provavelmente deve-se a sobreposição de picos através da maior energia requerida pelo sistema para que promovesse a fusão.

Para melhorar as características da mobilidade eletroforética é adicionado o dispersante de poliacrilato de sódio na suspensão, porém deve-se fazer a análise de potencial zeta para verificar a influêcia exata do dispersante na solução $2 \%$ em volume da amostra $30 \%$ em precursor. O gráfico da figura 4 e 5 mostra os resultados obtidos.

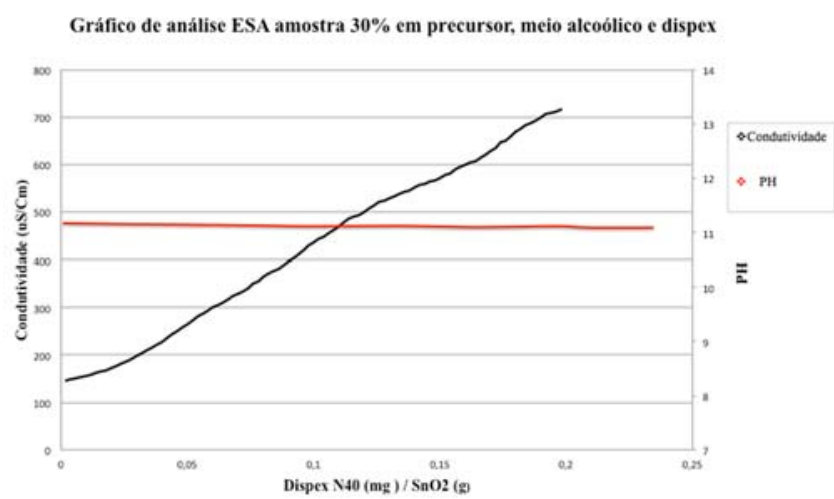

Figura 4: Variação do pH e da condutividade elétrica de uma dispersão de $\mathrm{SnO} 2$ (1\% em fração sólida) durante a adição de uma solução de dispex N40.

O gráfico da figura 4 nos mostra a variação de potencial zeta em função da adição de dispersante, essa que é intimamente ligada ao $\mathrm{pH}$, assim, o mecanismo de estabilização predominante é o estérico, uma vez que a reação do dispersante com a superfície da partícula gera apenas água, que não altera o pH da suspensão. A condutividade portanto é aumentada devido a solubilização desta na fase líquida uma vez que a superfície da partícula já esta saturada.

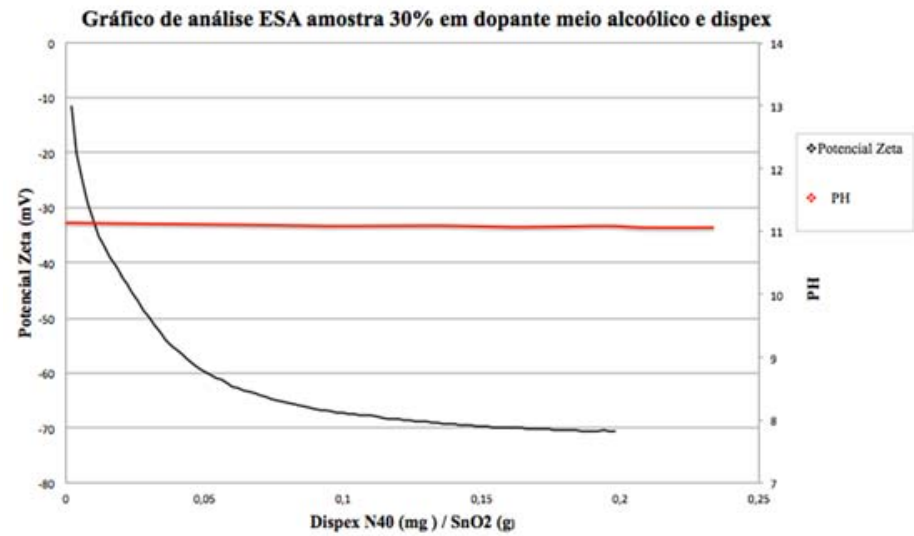

Figura 5: Variação do pH e do potêncial zeta de uma dispersão de $\mathrm{SnO} 2$ (1\% em fração sólida) durante a adição de uma solução de dispex N40.

A partir da figura 5 Observa-se que a estabilização da suspensão é controlada pelo potencial elétrico da superfície das partículas, oriunda da dupla camada elétrica formada pelos contra-íons, íons que apresentam cargas opostas àquela da 
superfície [4], o que permite concluir que o mecanismo de estabilização é eletroesterico.

A figura 6 mostra a análise de deposição eletroforética (EPD) da amostra de 30\% em precusor em meio alcoólico calcinada $2 \%$ em volume.

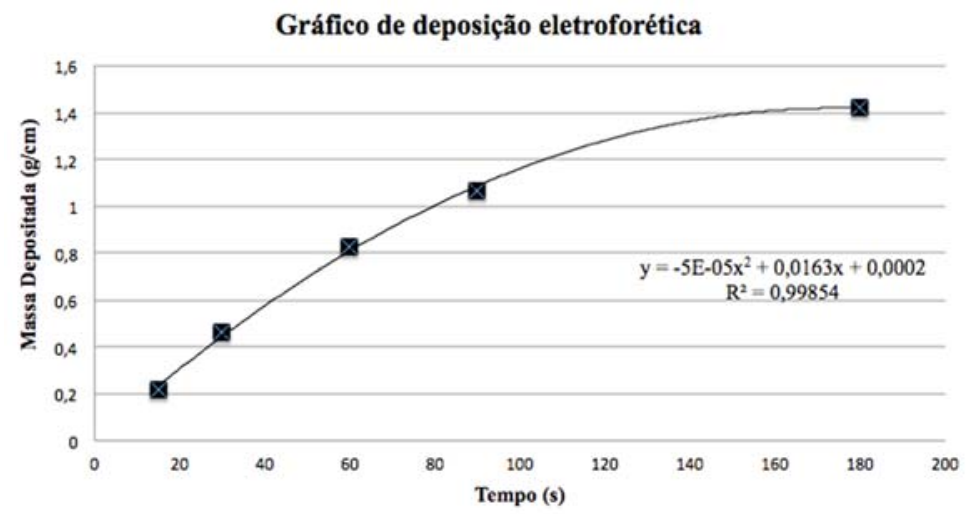

figura 6: Gráfico representativo do ensaio ESA onde se varia o tempo de permanecia da suspensão em contato com o eletrodo em função da massa depositada no eletrodo.

O valor médio de deposição de massa no eletrodo deferido pela equação de segundo grau no gráfico abaixo é de $1,13 \mathrm{~g} . \mathrm{s} / \mathrm{cm}$. É notório que nos instantes iniciais a deposição se dá a uma velocidade maior do que em períodos maiores, isso porque a medida que uma camada já é depositada, a interação entre o eletrodo e a suspensão é diminuída.

\section{CONCLUSÃO}

No trabalho apresentado foi feito uma sintetização de uma composição de óxido de estanho dopado em 30\% $\mathrm{Sr}_{2}+$ por meio do precursor nitrato de estrôncio. $\mathrm{O}$ processo utilizado foi através da moagem em meio alcoólico e calcinação á $700^{\circ} \mathrm{C}$ onde as fases formadas foram o estanato de estrôncio fase estável ( $\left.\mathrm{SrSnO}_{3}\right)$. Com a amostra calcinada foi feita uma suspensão aquosa com adição de dispersante a base de poliacrilato de sódio e submetida ao ensaio ESA que nos apresentou valores de $\mathrm{pH}$ constante igual a 11. Esse valor de $\mathrm{pH}$ possibilitou que o dispersante estéreo utilizado com suas características básicas estabilizasse a suspensão graças a adsorção deste nas superfícies das partículas, que no caso anterior a sintetização o óxido de estanho apresentava $\mathrm{pH}$ igual a 3,8 o que não o tornava possível.

Outro ponto observado foi que o $\mathrm{PH}$ se manteve constante á medida que se aumentava a condutividade em função da adição de dispersante, isso se deve ao fato de que a variação de potencial zeta se deve unicamente a adsorção do dispersante polimérico á superfície do material. Sendo assim podemos concluir que o dispersante se encontra protonado e ao se adsorver na superfície do material aumenta significativamente a carga superficial das partícula [5]. Como consequência aumenta-se também a mobilidade eletroforética o que torna possível a deposição eletroforética totalmente viável a esse tipo de material, que no caso era o objetivo do trabalho. Foi também medido o valor médio de deposição de massa em função do tempo na composição estudada e na tensão de $10 \mathrm{~V}$ no valor de $1,13 \mathrm{~g} . \mathrm{s} / \mathrm{cm}$ o que é considera significativa. 


\section{Agradecimentos}

Meu agradecimento especial ao Prof. Dr. Gilberto Pereira por me proporcionar essa experiência de aprofundamento em meus estudos de graduação, a sua disposição e preocupação com meu desempenho.

Agradeço também ao Centro Universitário da Fei que além de depositar sua confiança em seus alunos e funcionários os ajuda a concretizar sonhos e grandes realizações pessoais.

\section{REFERÊNCIAS}

1 Z. M. Jarzebski, Physical Properties Of SnO2 Materials, 1976, vol. 123, No. 10, pp. $333 \mathrm{C}$

2 NASCIMENTO, M.R, síntese e caracterização catalítica do sistema $\mathrm{Sr}_{1-\mathrm{x}} \mathrm{Nix}_{\mathrm{x}} \mathrm{SnO}_{3 .} 2007$ pp. $13,14,17,19$

3 Mineral Yearbook, 2007. Disponível em minerals.usgs.gov/minerals/pubs/ commodity/strontium/myb1-2007-stron.pdf, acessado em 23/06/2014

4 CALLISTER, W. D. Jr. Ciência e Engenharia de Materiais: Uma Introdução. $8^{\mathrm{a} e d i c ̧ a ̃ o . ~}$ Rio de Janeiro: LTC, 2012.

5 D. Gouvêa, B. B. S. Murad, Influência das características ácido-básicas da superfície dos óxidos na estabilidade de suspensões cerâmicas de $\mathrm{Al}_{2} \mathrm{O}_{3}$ ou $\mathrm{SnO}_{2}$ com dispersantes, disponível em: scielo.br/scielo.php?script=sci_arttext\&pid=S036669132001000100010. Acessado em 21/07/2014 